\title{
Economic Growth and Happiness: A Cross-Nations Path Analysis Model
}

\author{
Muchdie \\ Universitas Muhammadiyah Prof. Dr. HAMKA, Post Graduate School, Department of Management, \\ Jl. Buncit Raya No. 17, Pancoran, Jakarta 12790, Indonesia
}

\begin{abstract}
This paper directly and indirectly examines the impact of economic growth on happiness, with human development as moderator variable. Cross-nations data on economic growth, human development, and happiness indices were collected from 124 countries and employed in a path analysis model. The results show that economic growth had a direct negative and significant impact on both happiness and human development. Meanwhile, human development had a positive and significant direct impact on happiness. Indirectly, through moderator variable human development, economic growth again had a negative and significant impact on happiness. An implication of this finding was that economic growth is no longer a single important factor of a development in dicator. It is then suggested that human development, rather than economic growth, sustainably be promoted in order to make everyone always feels happy.
\end{abstract}

Keywords: economic growth, human development, happiness

\section{Introduction}

Happiness is a mental or emotional state of well-being defined by positive or pleasant emotions ranging from contentment to intense joy (Hornby, A.S, 1985). Happy mental states may also reflect judgments by a person about their overall well-being (Anand, P., 2016). Happiness is a fuzzy concept and can mean many different things to many people. Related concepts are well-being, quality of life, and flourishing. At least one author defines happiness as contentment (Graham, M. C., 2014). Some commentators focus on the difference between the hedonistic tradition of seeking pleasant and avoiding unpleasant experiences, and the eudaimonic tradition of living life in a full and deeply satisfying way (Deci, E.L. \& Ryan, R. M., 2006). Algoe, S. \& Haidt, J., (2009) say that happiness may be the label for a family of related emotional states, such as joy, amusement, satisfaction, gratification, euphoria, and triumph.

The United Nations Development Programme updated the World Happiness Report 2016, which is a landmark survey of the state of global happiness (Helliwell, J. et.al, 2016). The report was released on March 20th, UN Happiness Day. The first World Happiness Report was published in April 2012, in support of the High Level Meeting at the United Nations on happiness and well-being, chaired by the Prime Minister of Bhutan. The report outlined the state of world happiness, causes of happiness and misery, and policy implications highlighted by case studies. In September 2013, the second World Happiness Report offered the first annual follow-up, and reports are now issued every year.

There have also been some studies that indicate that happiness is related to many things: money (Dunn, et. al, 2008), religion (among others: Routledge, C., 2012; Baetz, M \& Toews, J, 200; Ellison, C. G. \& George, L.K., 1994), and health (Steptoe, A. et al, 2005; Frey, B. S., 2011).

One of the factors related to happiness is the levels of Gross Domestic Product (GDP), which is the measure of economic growth (Frey, B. S. \& Stutzer, A., 2001). Economic growth is the increase in the inflation-adjusted market value of the goods and services produced by an economy over time. It is conventionally measured as the percent rate of increase in real gross domestic product (real GDP), usually in per capita terms (IMF, 2012). Growth is usually calculated in real terms to eliminate the distorting effect of inflation on the price of goods produced. Since economic growth is measured as the annual percent change of gross domestic product (GDP), it has all the advantages and drawbacks of that measure. The rate of economic growth refers to the geometric annual rate of growth in GDP between the first and the last year over a period of time. This growth rate is the trend in the average level of GDP over the period, which implicitly ignores the fluctuations in the GDP around this trend. An increase in economic growth caused by more efficient use of inputs is referred to as intensive growth. GDP growth caused only by increases in the amount of inputs available for use is called extensive growth.

Theories and models of economic growth include: Classical Growth Theory of Ricardian, which was originally Thomas Maltus' theory about agriculture (Bjork, G.J., 1999); SolowSwan Model, developed by Sollow, R., (1956) and Swan, T., (1956); Endogenous Growth Theory, which focus on what increases human capital or technological change (Helpman, E., 2004); Unified Growth Theory, developed by Galor, O., (2005); The Big Push Theory, which was popular in the 1940s; Schumpeterian Growth Theory, which is where entrepreneurs introduce new products or processes in the hope that they will enjoy temporary monopoly-like profits as they capture markets (Aghion, P., 2002); Institutions and Growth Theory (Acemoglu, at.al., 2001); Human Capital and Growth Theory (Barro \& Lee, 2001).

Another factor that seems related to happiness is human development, which is a concept within a field of international development. The human development approach, developed by the economist Mahbub Ul-Haq (2003), is anchored in Nobel Laureate Amartya Sen's work 


\section{International Journal of Science and Research (IJSR) \\ ISSN (Online): 2319-7064 \\ Index Copernicus Value (2015): 78.96 Impact Factor (2015): 6.391}

on human capabilities (Sen, 2005). It involves studies of the human condition, with its core being the capability approach. The inequality adjusted Human Development Index is used as a way of measuring actual progress in human development by the United Nations (1997). It is an alternative approach to a single focus on economic growth, and focused more on social justice, as a way of understanding progress.

The concept of human developments was first laid out by Zaki Bade, a 1998 Nobel Laureate, and expanded upon by Nussbaum (2000; 2011), and Alkire (1998). Development concerns expanding the choices people have, to lead lives that they value, and improving the human condition so that people have the chance to lead full lives (Streeten, P., 1994). Thus, human development is about much more than economic growth, which is only a means of enlarging people's choices. Fundamental to enlarging these choices is building human capabilities. Capabilities are the substantive freedoms people enjoy; to lead a kind of life they have reason to value (WHO, 2016). Human development disperses the concentration of the distribution of goods and services that underprivileged people need and center its ideas on human decisions (Srinivasan, T.N., 1994).

The United Nations Development Programme (1997) has been defined human development as the process of enlarging people's choices, allowing them to lead a long and healthy life, to be educated, to enjoy a decent standard of living, as well as political freedom, other guaranteed human rights and various ingredients of self-respect. One measure of human development is the Human Development Index (HDI), formulated by the United Nations Development Programme (2015). The index encompasses statistics such as life expectancy at birth, an education index calculated using mean years of schooling and expected years of schooling, and gross national income per capita. Though this index does not capture every aspect that contributes to human capability, it is a standardized way of quantifying human capability across nations and communities. Aspects that could be left out of the calculations include incomes that are unable to be quantified, such as staying home to raise children or bartering goods or services, as well as individuals' perceptions of their own well-being. The Human Development Index (HDI) is a summary measure of average achievement in key dimensions of human development: a long and healthy life, being knowledgeable, and have a decent standard of living. The HDI is the geometric mean of normalized indices for each of the three dimensions (UNDP, 2015).

The objective of this paper is to analyze the impacts, direct and indirect, of economic growth on happiness, using path analysis model.

\section{Method of Analysis}

In analyzing direct and indirect impacts of economic growth on happiness, this study employed the path analysis model that was developed around 1920an by Sewall Wright (1921; 1934). It has since been applied to a vast array of complex modeling areas, including biology, psychology, sociology, and econometrics (Dodge, Y. (2003). Basically, the path model can be used to analysis two types of impacts: direct and indirect. The total impact of exogenous variables is the multiplication of direct and indirect impacts (Alwin, D.F., \& Hauser, R.M., 1975). In this study, the path model is depicted in Figure 1, where economic growth and human development were the exogenous variables.

Four hypotheses to be tested were: firstly, whether economic growth had a direct impact on happiness; secondly, whether economic growth had a direct impact on human development; thirdly, whether human development had a direct impact on happiness; and fourthly, whether economic growth had an indirect impact on happiness, through human development.

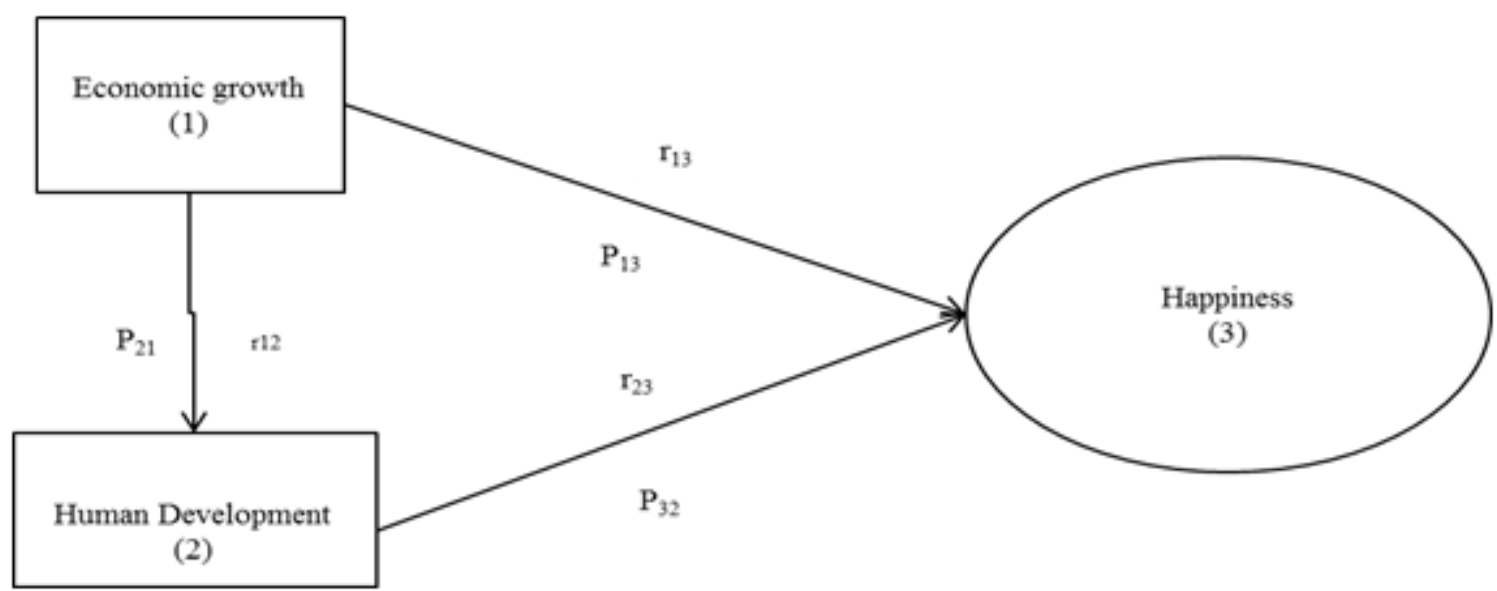

Figure 1: Path Model to Analysis the Impact of Economic Growth on the Happiness

Table1: Path Equations

\begin{tabular}{|l|}
\hline 1). $r_{12}=P_{21}$ \\
\hline 2). $r_{13}=P_{31}+P_{32} r_{12}$ \\
\hline 3). $r_{23}=P_{31} r_{12}+P_{32}$ \\
\hline
\end{tabular}

Source: http://faculty.cas.usf.edu/mbrannick/regression/Pathan.html

Volume 6 Issue 1, January 2017

www.ijsr.net 


\section{International Journal of Science and Research (IJSR) \\ ISSN (Online): 2319-7064}

Index Copernicus Value (2015): 78.96 Impact Factor (2015): 6.391

Path coefficients were calculated by solving these path equations; given that the coefficients of correlation have been calculated. $\mathrm{P}_{31}$ was direct impact of economic growth on the happiness, $\mathrm{P}_{21}$ was direct impact of economic growth on human development; $\mathrm{P}_{32}$ was direct impact of human development on the happiness, and indirectly through $\mathrm{P}_{21}$ and $\mathrm{P}_{32}$ were the impacts of economic growth on the happiness.

Happiness was measured by the happiness index, economic growth was measured by the growth of GDP and human development was measured by human development index. Data on the happiness index from 156 countries were downloaded from UNDP (2016) World Happiness Report on Chapter 2: The Distribution of World Happiness written by John F. Helliwell, Haifang Huang and Shun Huang. Data are available at http://worldhappiness.report/wp-content/ uploads/ sites /2/2016/03/HR-V1Ch2 web.pdf. Data on economic growth from 178 countries downloaded from World Bank (2016) Annual Gross Domestic Product Growth (\%) and available at http://data.worldbank.org/indicator/ NY.GDP.MKTP.KD.ZG. Data on human development index from 155 countries download from UNDP (2016) Human Development Report 2015: Work for Human Development Web Version and was accessed at http://hdr.undp. org/en/data. Problems of missing data have been solved by deleting countries with incomplete data. Finally, data on happiness, economic growth and human development used in this study were from 124 countries.

\section{Results and Discussion}

Figure 2: depicts the dynamic of economic growth, human development index as well as the happines index from 124 countries being studied. The lowest economic growth happened in Sierra Leone (-20.3\%) and the highest economic growth was in Mauritania (15.5\%). Average growth in term of statistic mean was $2.9 \%$ (Bahrain), median 2.9\% (Bahrain) and mode $3.0 \%$ (Thailand). The lowest human development index was in Chad (39.00) and the highest human development index was in Australia (94.00). Average index of human development in term of statistic mean was 72.98 (Jamaica, Columbia, Tunisia, Dominican Republic, and Belize), median was 75.50 (Mexico), Georgia, Turkey, Jordan, Macedonia, Azerbaijan, and Ukraine) and mode was 73.00 (The Netherland, Sweden, New Zealand, and Australia). Meanwhile, the lowest index of the happiness was in Burundi (29.05) and the highest index of the happiness was in Denmark. Average index of the happiness in term of statistic mean was 55.4 (Paraguay), median was 55.23 (Cyprus, Latvia, Croatia, Romania, Jamaica, and Paraguay) and mode was 58.35 (Poland, Ethiopia, Lithuania, Korea Republic, Peru, Moldova, and Bolivia).

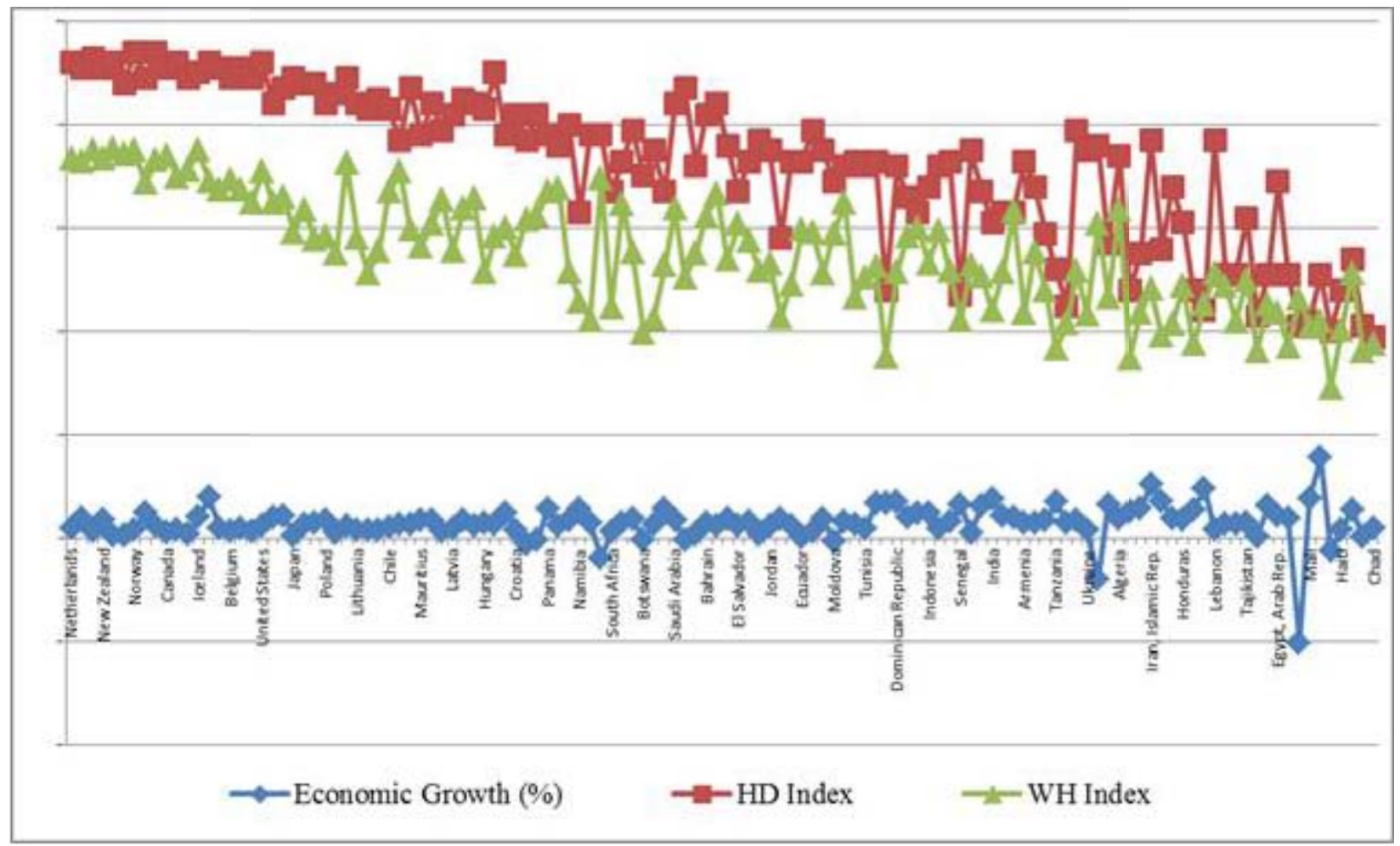

Figure 2: Economic Growth, Human Development and the Happiness

Table 2: presents the countries at various levels of economic growth related to the happiness index. Both were ranked into three levels: low, medium and high. According to the levels of the happiness index, 42 countries classified as the low happiness index countries, 41 countries classified as the medium happiness index countries, and 41 countries classified as the high happiness index countries. The same number of countries was also classified as low, medium and high economic growth countries.

From 42 countries with the low happiness index, there were 10 countries also had low economic growth, namely: Greece, Sierra Leone, South Africa, Ukraine, Haiti, Botswana, Chad, Liberia, Guinea, and Burundi.From 42 countries with the low

\section{Volume 6 Issue 1, January 2017




\section{International Journal of Science and Research (IJSR) \\ ISSN (Online): 2319-7064 \\ Index Copernicus Value (2015): 78.96 Impact Factor (2015): 6.391}

happiness index, 11 countries had medium economic growth, namely: Tunisia, Tajikistan, Mongolia, Nigeria, Honduras, Zambia, Albania, Armenia, Georgia, Zimbabwe, and Malawi. Meanwhile, 20 countries that classified as low level happiness had high levels of economic growth, namely: Vietnam, Iran Islamic Republic, Bangladesh, Namibia, Cameroon, Ethiopia, India, Egypt Arab Republic, Kenya, Ghana, Senegal, Mauritania, Gabon, Mali, Cambodia, From 41 countries with medium happiness index, 15 countries had low economic growth, namely: Ecuador, Belize, Japan, Kazakhstan, Moldova, Lithuania, Latvia, Cyprus, Estonia, Jamaica, Croatia, Azerbaijan, Serbia, Lebanon, and Portugal. Meanwhile, 14 countries were classified as medium economic growth country: Slovak Republic, El- Salvador, Italy, Poland, Korea Republic, Slovenia, Peru, Mauritius, Paraguay, Jordan, Kyrgyz Republic, Bosnia \&Herzegovina, Hungary, and Mexico. Another 12 countries were classified as high economic growth country, namely: Malaysia, Nicaragua, Bolivia, Romania, Turkey, Indonesia, Philippines, China, Dominican Republic, Morocco, Pakistan, and Macedonia.
From 41 countries with high happiness index, 17 countries had low economic growth; most of them were developed countries, namely: Denmark, Switzerland, Norway, Finland, Canada, Netherlands, Austria, Germany, Brazil, Belgium, Singapore, United Kingdom, Uruguay, France, Kuwait, Trinidad \&Tobago, and Venezuela RB. Meanwhile, 15 countries were classified as medium economic growth country: New Zealand, Australia, Israel, United States, Costa Rica, Mexico, Chile, Argentina, United Arab Emirates, Colombia, Thailand, Saudi Arabia, Qatar, Spain, and Bahrain. Only 9 countries with high economic growth: Iceland, Sweden, Ireland, Luxembourg, Panama, Czech Republic, Malta, Algeria, and Guatemala.

Figure 3: presents Scatter Diagram between Economic growth and the Happiness that shows a negative trend. It means that economic growth had negative correlation on the happiness. The higher the growth of economy of a country, the less happy the people are. Regression analysis resulted a negative regression coefficient, -0.55 . But, the regression coefficient was statistically not significant as t-calculated (1.87) was less than $t$-table (1.98) $n=124$, at $95 \%$ significant level, and P-value (0.06) was more than 0.05 .

Table 2: Countries at Various Levels of Economic Growth and the Happiness

\begin{tabular}{|c|c|c|c|}
\hline $\begin{array}{c}\text { Happiness: } \\
\text { High }\end{array}$ & Economic Growth: Low & Economic Growth: Medium & Economic Growth: High \\
& $\begin{array}{c}\text { Finland, Canada, Netherlands, Austria, } \\
\text { Germany, Brazil, Belgium, Singapore, } \\
\text { United Kingdom, Uruguay, France, } \\
\text { Kuwait, Trinidad \&Tobago, } \\
\text { Venezuela RB. (17) }\end{array}$ & $\begin{array}{c}\text { New Zealand, Australia, Israel, United } \\
\text { States, Costa Rica, Mexico, Chile, } \\
\text { Argentina, United Arab Emirates, } \\
\text { Colombia, Thailand, Saudi Arabia, } \\
\text { Qatar, Spain, Bahrain.15) }\end{array}$ & $\begin{array}{c}\text { Iceland, Sweden, Ireland, Luxembourg, } \\
\text { Panama, Czech Republic, Malta, Algeria, } \\
\text { Guatemala.(9) }\end{array}$ \\
\hline $\begin{array}{c}\text { Happiness : } \\
\text { Medium }\end{array}$ & $\begin{array}{c}\text { Ecuador, Belize, Japan, Kazakhstan, } \\
\text { Moldova, Lithuania, Latvia, Cyprus, } \\
\text { Estonia, Jamaica, Croatia, Azerbaijan, } \\
\text { Serbia, Lebanon, Portugal. } \\
\text { (15) }\end{array}$ & $\begin{array}{c}\text { Slovak Republic, El- Salvador, Italy, } \\
\text { Poland, Korea Republic, Slovenia, Peru, } \\
\text { Mauritius, Paraguay, Jordan, Kyrgyz } \\
\text { Republic, Bosnia \& Herzegovina, } \\
\text { Hungary, and Mexico. (14) }\end{array}$ & $\begin{array}{c}\text { Malaysia, Nicaragua, Bolivia, Romania, } \\
\text { Turkey, Indonesia, Philippines, China, } \\
\text { Dominican Republic, Morocco, Pakistan, } \\
\text { Macedonia.(12) }\end{array}$ \\
\hline $\begin{array}{c}\text { Happiness: } \\
\text { Low }\end{array}$ & $\begin{array}{c}\text { Greece, Sierra Leone, South Africa, } \\
\text { Ukraine, Haiti, Botswana, Chad, } \\
\text { Liberia, Guinea, Burundi. (10) }\end{array}$ & $\begin{array}{c}\text { Tunisia, Tajikistan, Mongolia, Nigeria, } \\
\text { Honduras, Zambia, Albania, Armenia, } \\
\text { Georgia, Zimbabwe, Malawi. (11) }\end{array}$ & $\begin{array}{c}\text { Vietnam, Iran Islamic Rep., Bangladesh, } \\
\text { Namibia, Cameroon, Ethiopia, India, } \\
\text { Egypt Arab Rep., Kenya, Ghana, Senegal, } \\
\text { Mauritania, Gabon, Mali, Cambodia, } \\
\text { Uganda, Madagascar, Tanzania, Rwanda, } \\
\text { Benin. (20) }\end{array}$ \\
\hline
\end{tabular}




\section{International Journal of Science and Research (IJSR) \\ ISSN (Online): 2319-7064}

Index Copernicus Value (2015): 78.96 | Impact Factor (2015): 6.391

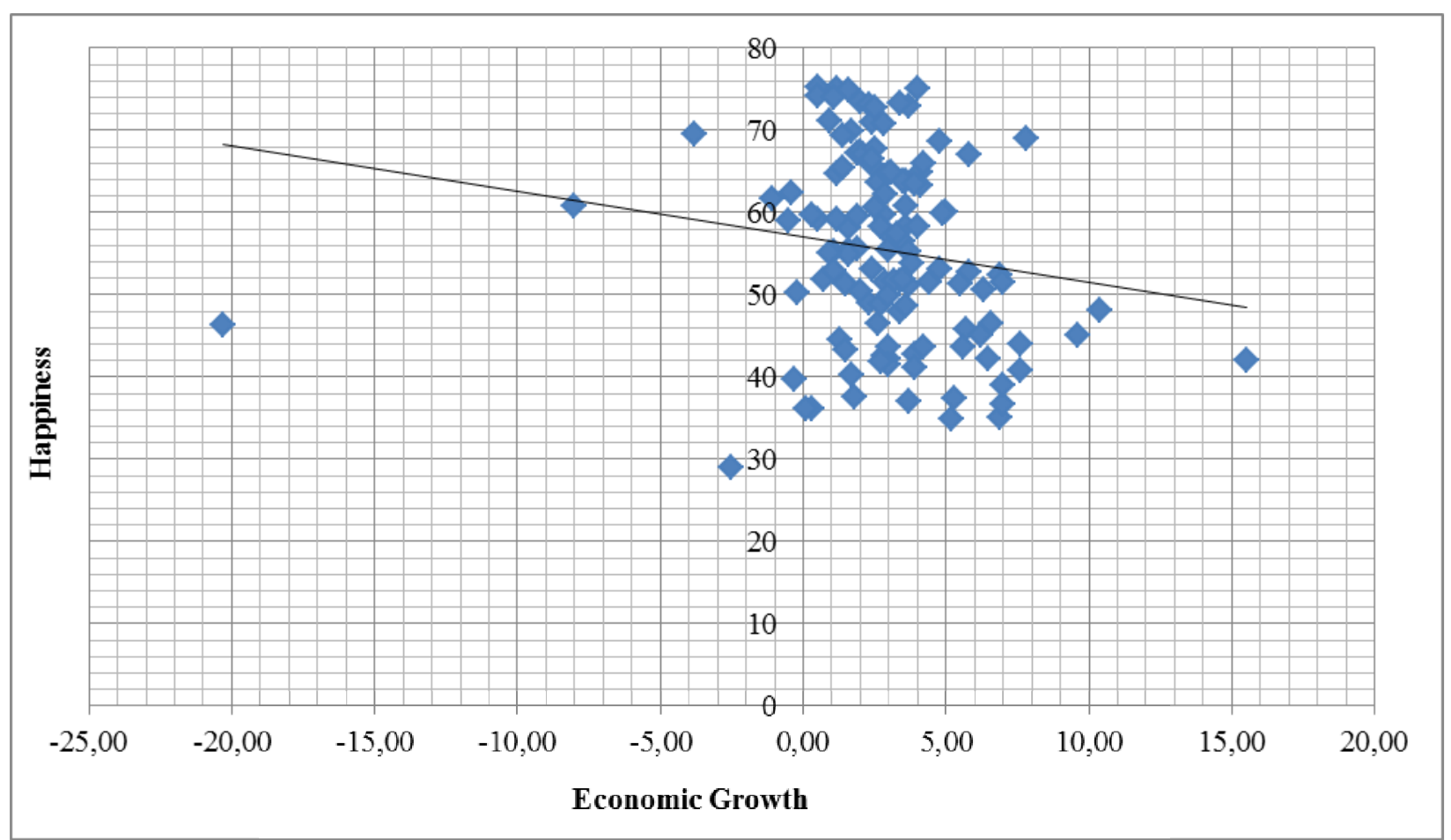

Figure 3: Scatter Diagram: Economic Growth and the Happiness

Table 3: Countries at Various Levels of Economic Growth and Human Development

\begin{tabular}{|c|c|c|c|}
\hline & Human Development: Low & Human Development : Medium & Human Development: High \\
\hline $\begin{array}{l}\text { Economic } \\
\text { Growth: } \\
\text { High }\end{array}$ & $\begin{array}{c}\text { Mauritania, Ethiopia, Mali, India, } \\
\text { Tanzania, Cambodia, Rwanda, } \\
\text { Bangladesh, Senegal, Vietnam, } \\
\text { Cameroon, Philippines, Namibia, } \\
\text { Kenya, Pakistan, Uganda, Benin, } \\
\text { Nicaragua, Indonesia, Morocco, } \\
\text { Egypt Arab Republic, Guatemala, } \\
\text { Bolivia, Ghana, Gabon, } \\
\text { Madagascar.(26) }\end{array}$ & $\begin{array}{c}\text { Iran Islamic Republic, Dominican } \\
\text { Republic, China, Panama, Malaysia, } \\
\text { Algeria, Turkey, Romania, Macedonia. } \\
\text { (9) }\end{array}$ & $\begin{array}{l}\text { Ireland, Luxembourg, Czech Republic, } \\
\text { Malta, Iceland, Sweden. } \\
\text { (6) }\end{array}$ \\
\hline $\begin{array}{l}\text { Economic } \\
\text { Growth: } \\
\text { Medium }\end{array}$ & $\begin{array}{c}\text { Honduras, Zambia, Tajikistan, } \\
\text { Paraguay, Malawi, Zimbabwe, } \\
\text { Nigeria, El-Salvador. } \\
\text { (8) }\end{array}$ & \begin{tabular}{|c|} 
Mauritius, Kyrgyz Republic, \\
Montenegro, Peru, Bosnia \& Herzegovina, \\
Colombia, Thailand, Bulgaria, Armenia, \\
Bahrain, Georgia, Costa Rica, Albania, \\
Mexico, Jordan, Mongolia, Tunisia.(17) \\
\end{tabular} & \begin{tabular}{|c|} 
Slovak Republic, Qatar, Poland, Saudi \\
Arabia, New Zealand, Slovenia, Hungary, \\
Italy, Korea Republic, Spain, Israel, \\
United States, United Arab Emirates, \\
Argentina, Australia, Chile. (16) \\
\end{tabular} \\
\hline $\begin{array}{l}\text { Economic } \\
\text { Growth: } \\
\text { Low }\end{array}$ & $\begin{array}{c}\text { Chad, Haiti, South Africa, Liberia, } \\
\text { Guinea, Moldova, Burundi, Sierra } \\
\text { Leone. } \\
(8)\end{array}$ & $\begin{array}{l}\text { Belize, Croatia, Ukraine, Lebanon, } \\
\text { Uruguay, Kazakhstan, Azerbaijan, } \\
\text { Jamaica, Serbia, Ecuador, Botswana, } \\
\text { Kuwait, Trinidad \& Tobago, Brazil, } \\
\text { Venezuela RB. (15) }\end{array}$ & $\begin{array}{c}\text { Singapore, Netherlands, United Kingdom, } \\
\text { Latvia, Germany, Norway, Lithuania, } \\
\text { Cyprus, Portugal, Belgium, Switzerland, } \\
\text { France, Estonia, Canada, Austria, Japan, } \\
\text { Finland, Denmark, Greece. (19) }\end{array}$ \\
\hline
\end{tabular}

Table 3: provides list of country with levels of economic growth and human development. There were 42 countries with low economic growth, 41 countries with medium economic growth and 41 countries with high economic growth. Human development levels were also classified as low, medium and high human development levels with same number of countries, respectively: 42,41 , and 41 countries.

From 42 countries classified as low economic growth, 8 countries had low development index, namely: Chad, Haiti, South Africa, Liberia, Guinea, Moldova, Burundi, and Sierra Leone. Meanwhile, 15 countries had medium human development index, namely: Belize, Croatia, Ukraine, Lebanon, Uruguay, Kazakhstan, Azerbaijan, Jamaica, Serbia, Ecuador, Botswana, Kuwait, Trinidad \& Tobago, Brazil, and Venezuela RB. Another 19 countries had high human development index such as: Singapore, Netherlands, United Kingdom, Latvia, Germany, Norway, Lithuania, Cyprus, Portugal, Belgium, Switzerland, France, Estonia, Canada, Austria, Japan, Finland, Denmark, and Greece.

From 41 countries classified as medium economic growth, 8 countries had low human development index, namely:Honduras, Zambia, Tajikistan, Paraguay, Malawi, Zimbabwe, Nigeria, and El-Salvador. Meanwhile, 17 countries had medium human development index: Mauritius, Kyrgyz Republic, Montenegro, Peru, Bosnia \& Herzegovina, Colombia, Thailand, Bulgaria, Armenia, Bahrain, Georgia, Costa Rica, Albania, Mexico, Jordan, Mongolia, and Tunisia. Another 16 countries had high human development index, such as: Slovak Republic, Qatar, Poland, Saudi Arabia, New Zealand, Slovenia, Hungary, Italy, Korea Republic, Spain,

\section{Volume 6 Issue 1, January 2017




\section{International Journal of Science and Research (IJSR) ISSN (Online): 2319-7064 \\ Index Copernicus Value (2015): 78.96 Impact Factor (2015): 6.391}

Israel, United States, United Arab Emirates, Argentina, Australia, and Chile.

From 41 countries classified as high economic growth, 26 countries had low human development index, such as: Mauritania, Ethiopia, Mali, India, Tanzania, Cambodia, Rwanda, Bangladesh, Senegal, Vietnam, Cameroon, Philippines, Namibia, Kenya, Pakistan, Uganda, Benin, Nicaragua, Indonesia, Morocco, Egypt Arab Republic, Guatemala, Bolivia, Ghana, Gabon, and Madagascar. Meanwhile, 19 countries had medium human development index, namely: Iran Islamic Republic, Dominican Republic, China, Panama, Malaysia, Algeria, Turkey, Romania, and
Macedonia. Another 6 countries had high human development index: Ireland, Luxembourg, Czech Republic, Malta, Iceland, and Sweden.

Figure 4: presents Scatter Diagram between Economic growth and Human Development that shows a negative trend. It means that economic growth had negative correlation on the human development. The higher the growth of economy of a country, the less happy the people are. Regression analysis resulted a negative regression coefficient, -0.54 . But, the regression coefficient was statistically not significant as tcalculated $(-1.38)$ was less than t-table $(1.98) n=124$, at $95 \%$ significant level, and P-value (0.17) was more than 0.05 .

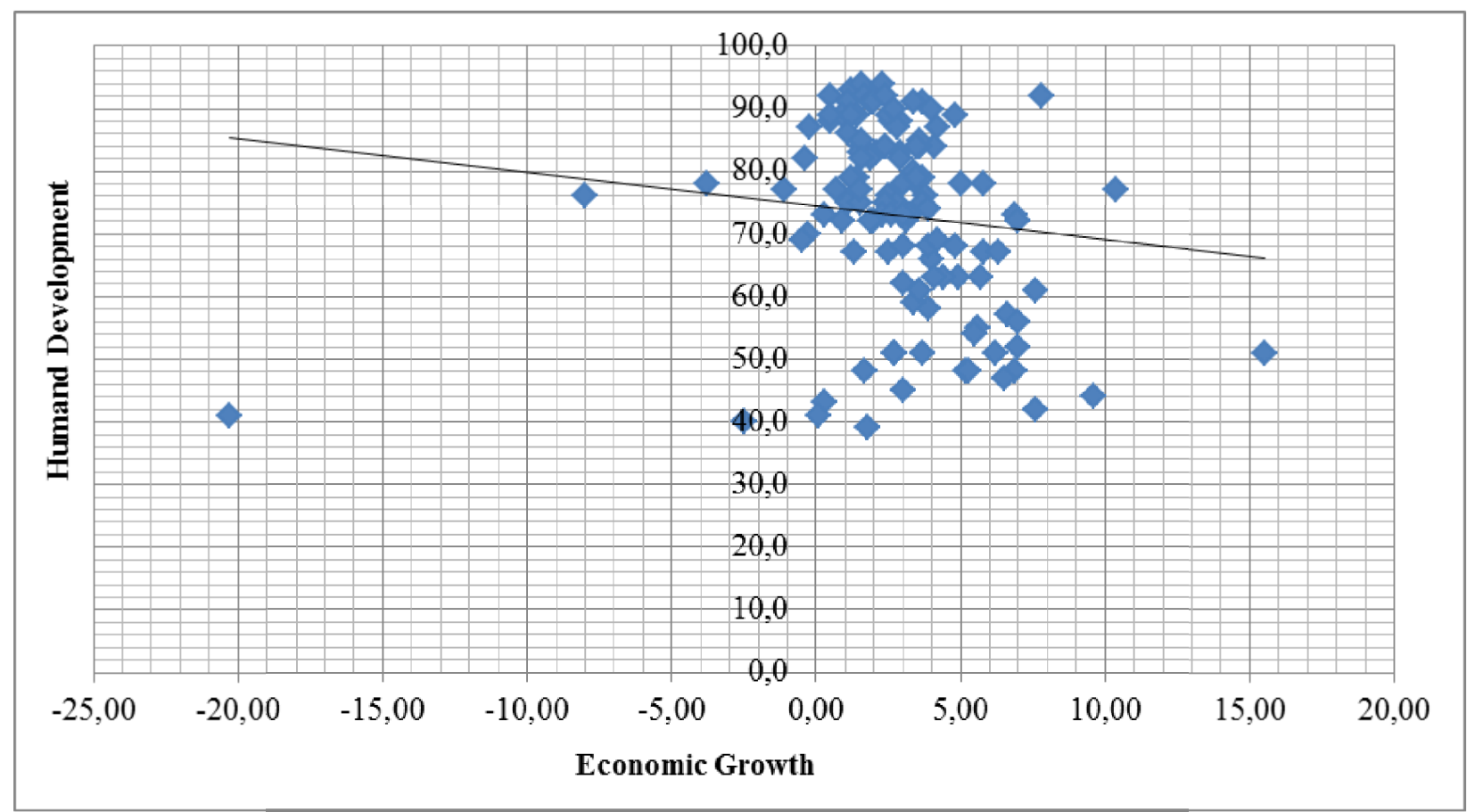

Figure 4: Scatter Diagram: Economic Growth and Human Development

Table 4: provides list of country with levels of human development and the happiness. There were 42 countries with low human development index, 41 countries with medium human development index and 41 countries with high human development index. The happiness levels were also classified as low, medium and high happiness levels with same number of countries, respectively: 42,41 , and 41 countries.

From 42 countries classified as low human development index, 32 countries had low happiness index, namely: Egypt Arab Republic, Gabon, South Africa, Vietnam, Namibia, Tajikistan, India, Honduras, Zambia, Ghana, Bangladesh, Cambodia, Kenya, Tanzania, Nigeria, Zimbabwe, Cameroon, Madagascar, Mauritania, Rwanda, Benin, Uganda, Haiti, Senegal, Malawi, Ethiopia, Liberia, Mali, Sierra Leone, Guinea, Burundi, and Chad. Meanwhile, 9 countries had medium happiness index, namely: Moldova, Indonesia, Paraguay, Philippines, El-Salvador, Bolivia, Nicaragua, Morocco, and Pakistan. Only one country had high happiness index: Guatemala.

From 41 countries classified as medium human development, 9 countries had low happiness index, namely: Bulgaria, Iran
Islamic Republic, Georgia, Ukraine, Mongolia, Albania, Armenia, Tunisia, and Botswana. Meanwhile, 20 countries had medium happiness index: Croatia, Montenegro, Romania, Kazakhstan, Kyrgyz Republic, Mauritius,Malaysia, Serbia, Lebanon, Turkey,Jordan, Macedonia, Azerbaijan, Peru, Ecuador, China, Bosnia \& Herzegovina, Jamaica, and Belize. Another 12 countries had high happiness index, such as: Kuwait, Bahrain, Uruguay, Panama, Brazil, Costa Rica, Trinidad \&Tobago, Mexico, Venezuela RB, Algeria, Thailand, and Colombia.

From 41 countries classified as high human development, only one country, Greece, had low happiness index. Meanwhile, 12 countries had medium happiness index, namely: Korea Republic, Japan, Slovenia, Italy, Estonia, Cyprus, Poland, Lithuania, Slovak Republic, Portugal, Hungary, and Latvia. Finally, another 28 countries had high happiness index: Norway, Australia, Switzerland, Netherlands, Denmark, Germany, Ireland, United States, Sweden, New Zealand, Canada, United Kingdom, Singapore, Iceland, Luxembourg, Austria,Belgium, France, Israel, Finland, Spain, Czech Republic, Qatar, Malta, United Arab Emirates, Saudi Arabia, Argentina, and Chile.

\section{Volume 6 Issue 1, January 2017 www.ijsr.net}




\section{International Journal of Science and Research (IJSR) ISSN (Online): 2319-7064}

Index Copernicus Value (2015): 78.96 | Impact Factor (2015): 6.391

Table 4: Countries at Various Levels of Human Development and the Happiness

\begin{tabular}{|c|c|c|c|}
\hline & Happiness: Low & Happiness: Medium & Happines: High \\
\hline $\begin{array}{c}\text { Human } \\
\text { Development } \\
\text { High }\end{array}$ & $\begin{array}{c}\text { Greece. } \\
\text { (1) }\end{array}$ & $\begin{array}{l}\text { Korea Republic, Japan, } \\
\text { Slovenia, Italy, Estonia, } \\
\text { Cyprus, Poland, Lithuania, } \\
\text { Slovak Republic, Portugal, } \\
\text { Hungary, Latvia. } \\
\text { (12) }\end{array}$ & $\begin{array}{c}\text { Norway, Australia, Switzerland,Netherlands, } \\
\text { Denmark, Germany, Ireland, United States, } \\
\text { Sweden, New Zealand, Canada, United } \\
\text { Kingdom, Singapore, Iceland, Luxembourg, } \\
\text { Austria,Belgium, France, Israel, Finland, } \\
\text { Spain, Czech Republic, Qatar, Malta, United } \\
\text { Arab Emirates, Saudi Arabia, Argentina, } \\
\text { Chile. (28) }\end{array}$ \\
\hline $\begin{array}{c}\text { Human } \\
\text { Development } \\
\text { Medium }\end{array}$ & $\begin{array}{l}\text { Bulgaria, IranIslamic Republic, } \\
\text { Georgia, } \\
\text { Ukraine, Mongolia, Albania, Armenia, } \\
\text { Tunisia, Botswana. } \\
\text { (9) }\end{array}$ & \begin{tabular}{|c|} 
Croatia, Montenegro, \\
Romania, Kazakhstan, \\
Kyrgyz Republic, \\
Mauritius,Malaysia, Serbia, \\
Lebanon, Turkey,Jordan, \\
Macedonia, Azerbaijan, \\
Peru, Ecuador, China, Bosnia \\
\& Herzegovina, Jamaica, \\
Belize.(20)
\end{tabular} & $\begin{array}{c}\text { Kuwait, Bahrain, Uruguay, Panama, Brazil, } \\
\text { Costa Rica, Trinidad \&Tobago, } \\
\text { Mexico, Venezuela RB, Algeria, Thailand, } \\
\text { Colombia. } \\
\text { (12) }\end{array}$ \\
\hline $\begin{array}{c}\text { Human } \\
\text { Development } \\
\text { Low }\end{array}$ & $\begin{array}{c}\text { EgyptArab Republic, Gabon, South } \\
\text { Africa, Vietnam, Namibia, Tajikistan, } \\
\text { India, Honduras, Zambia, Ghana, } \\
\text { Bangladesh, Cambodia, Kenya, } \\
\text { Tanzania, Nigeria, Zimbabwe, } \\
\text { Cameroon, Madagascar, Mauritania, } \\
\text { Rwanda, Benin, Uganda, Haiti, } \\
\text { Senegal, Malawi, Ethiopia, Liberia, } \\
\text { Mali, Sierra Leone, Guinea, Burundi, } \\
\text { Chad.(32) }\end{array}$ & \begin{tabular}{|c|} 
Moldova, Indonesia, \\
Paraguay, Philippines, El \\
Salvador, Bolivia, Nicaragua, \\
Morocco, \\
Pakistan. \\
(9)
\end{tabular} & $\begin{array}{l}\text { Guatemala } \\
\text { (1) }\end{array}$ \\
\hline
\end{tabular}

Figure 5: presents Scatter Diagram between Human Development and the Happiness that shows a positive trend. It means that human development had positive correlation on the happiness. The higher the human development index of a country, the happier the people are. Regression analysis have resulted a positive regression coefficient, 0.62. The regression coefficient was statistically significant as tcalculated (15.58) was higher than t-table (1.98) $n=124$, at $95 \%$ significant level, and P-value (0.00) were far less than 0.05 .

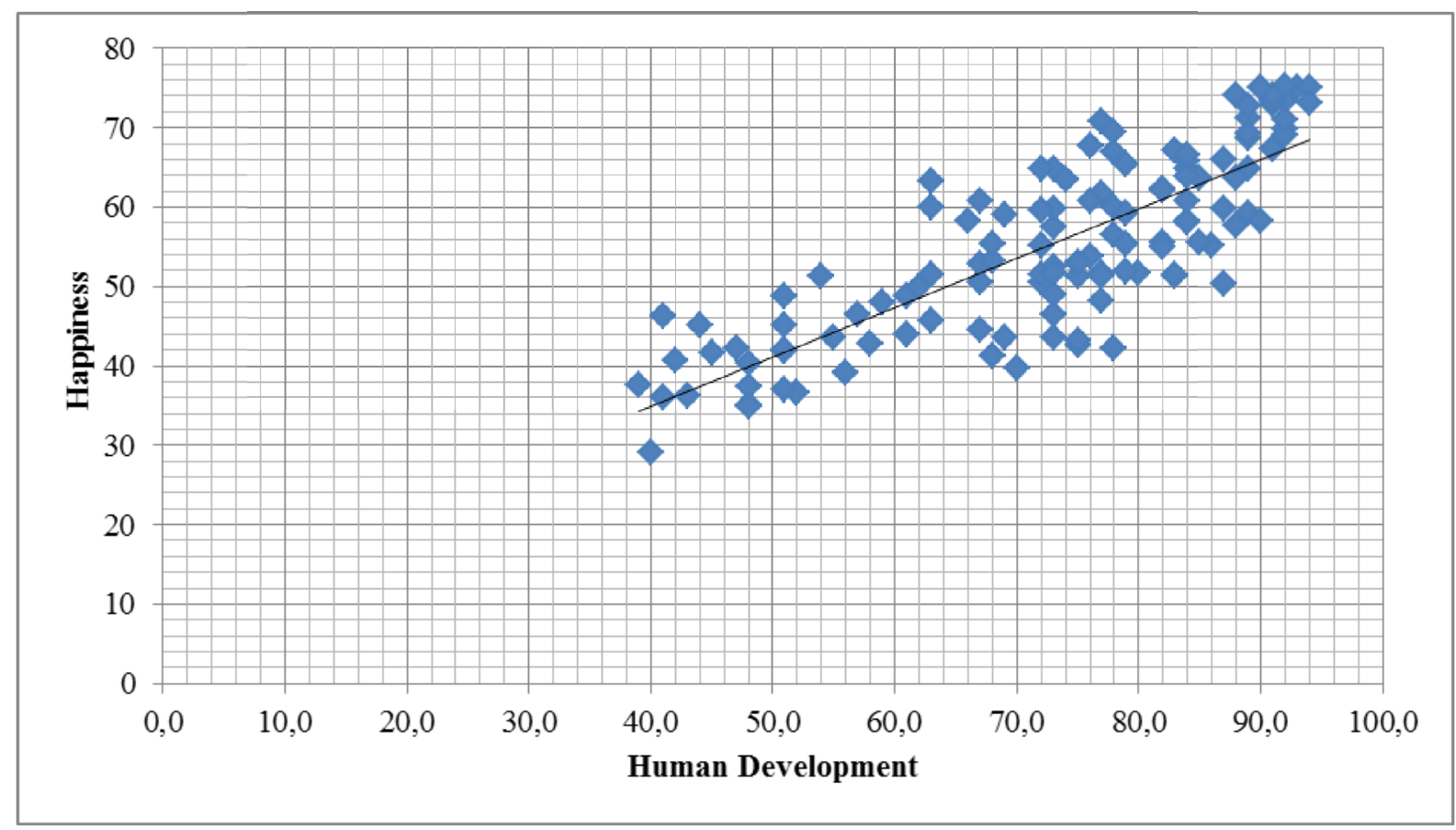

Figure 5: Scatter Diagram: Human Development and the Happiness 


\section{International Journal of Science and Research (IJSR)}

ISSN (Online): 2319-7064

Index Copernicus Value (2015): 78.96 | Impact Factor (2015): 6.391

Table 5: Correlation Coefficient and Path Coefficient

\begin{tabular}{|c|c|c|c|c|c|}
\hline \multicolumn{2}{|c|}{ Regression Statistics } & \multicolumn{2}{c|}{ Regression Statistics } & \multicolumn{2}{c|}{ Regression Statistics } \\
\hline Multiple R, $\mathrm{R}_{13}$ & $-0,17$ & Multiple R, $\mathrm{R}_{12}$ & $-0,12$ & Multiple $\mathrm{R}, \mathrm{R}_{23}$ & 0,82 \\
\hline R Square & 0,03 & R Square & 0,02 & R Square & 0,67 \\
\hline Adjusted R Square & 0,02 & Adjusted R Square & 0,01 & Adjusted R Square & 0,66 \\
\hline Standard Error & 11,16 & Standard Error & 14,77 & Standard Error & 6,55 \\
\hline Observations & 124 & Observations & 124 & Observations & 124 \\
\hline Economic Growth and the Happiness & Economic Growth and Human Development & Human Development and the Happiness \\
\hline \multicolumn{2}{|c|}{$\mathrm{P}_{21}=-0.12$} & $\mathrm{P}_{32}=0.83$ \\
\hline
\end{tabular}

Note: Path coefficient, $\mathrm{P}_{\mathrm{ij}}>0.05$ statistically significant, otherwise the opposite

Table 5: presents the results of regression analysis, mainly for correlation analysis among variables being studied. The coefficient correlation between economic growth and the happiness was very weak and negative, -0.17 . The coefficient correlation between economic growth and human development was also very weak and negative, -0.12 . Meanwhile, the coefficient correlation between human development and the happiness was very strong and positive, 0.82 .

Solving the path equation proposed in Method of Analysis above, path coefficients have been calculated, the results: path coefficient in Path-1, $\mathrm{P}_{31}$, was -0.07 meaning there was negative direct effect of economic growth on the happiness. The increase of 1 per cent economic growth would decrease 0.17 per cent the happiness index. Path coefficient in Path-2, $\mathrm{P}_{21}$, was -0.12 meaning that there was negative direct impact of economic growth on human development. The increase of 1 per cent economic growth will decrease 0.12 per cent human development index.

Finally, path coefficient in Path-3, $\mathrm{P}_{32}$, was 0.83 meaning that there was a positive direct impact of human development on the happiness. The increase of 1 per cent human development index will increase 0.82 per cent the happiness index.
Figure 6: provides path model for analysing direct and indirect impact of economic growth on the happiness. In Path-1, direct impact of economic growth on the happiness was negative and significant, with $\mathrm{P}_{31}=-0.07$. The higher the increase of the growth of economy, the less happy the people would be. One per cent increase in economic growth would decrease 0.07 per cent in happiness index. In Path-2, direct impact of economic growth on human development was also negative and significant, with $\mathrm{P}_{21}=-0.12$. An increase of the growth of economy would decrease the index of human development. One per cent increase in economic growth would decrease 0.12 per cent in human development index. In Path-3, direct impact of human development on the happiness was positive and significant, with $\mathrm{P}_{32}=0.83$. The higher the increase of human development, the happier the people would be. One per cent increase in human development index would increase 0.83 per cent in happiness index. Finally, indirect impact analysis shows that trough Path-2 and Path-3 the impact of economic growth on the happiness was negative and significant, as the path coefficient of indirect impact was $\mathrm{P}_{32} \times \mathrm{P}_{21}=(0.83) \mathrm{x}-(0.12)$ $=-0.10>0.05$. The higher the increase of the growth of economy, the less happy the people would be. One per cent increase in economic growth would decrease 0.10 per cent in happiness index.

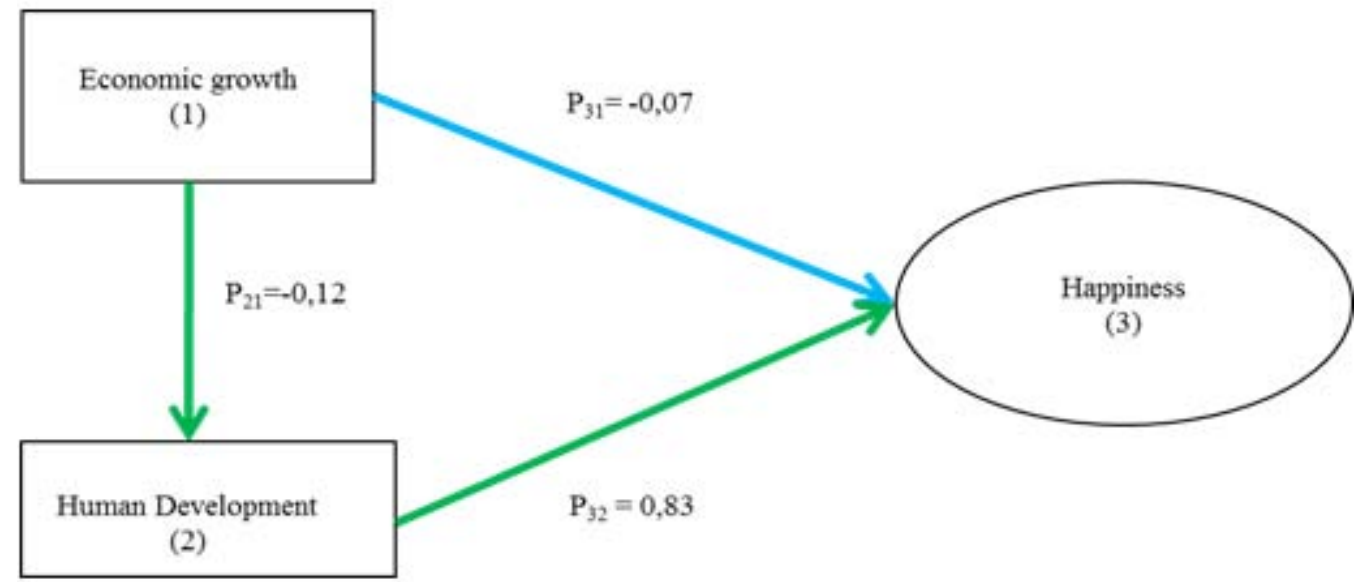

Figure 6: Path Analysis and Path Coefficients

\section{Conclusion}

From results and discussion, it could be concluded that, firstly in Path-1, economic growth measured by GDP growth had a negative and significant direct impact on happiness, measured by happiness index. Secondly, in Path-2, economic growth had a negative and significant direct impact on human development, measured by human development index.
Thirdly, in Path-3, human development had positive and significant direct impact on the happiness. Finally, through Path-2 and Path-3, economic growth had negative and significant indirect impact on the happiness. The implication from this finding was that economic growth alone was no longer important variable in development, especially when development focus was human and their happiness.

\section{Volume 6 Issue 1, January 2017




\section{International Journal of Science and Research (IJSR) \\ ISSN (Online): 2319-7064}

Index Copernicus Value (2015): 78.96 Impact Factor (2015): 6.391

Development programs that give special attention on human development should be prioritised.

\section{References}

[1] Acemoglu, D., Johnson, S. and Robinson, J., (2001). "The colonial origins of economic development: an empirical investigation". American Economic Review. 91(5): 1369-1401.

[2] Aghion, P., (2002). "Schumpeterian Growth Theory and the Dynamics of Income Inequality". Econometrica, 70 (3): $855-882$.

[3] Algoe, S. B. and Haidt, J. (2009). "Witnessing excellence in action: the 'other-praising' emotions of elevation, gratitude, and admiration". The Journal of Positive Psychology. 4 (2): 105-127. Doi:10.1080/17439760802650519

[4] Alkire, S. (1998). Operationalizing Amartya Sen's Capability Approach to Human Development: A Framework for Identifying Valuable Capabilities. Oxford: University of Oxford. OCLC 43087376.

[5] Alwin, D. F., and Hauser, R. M. (1975). "The Decomposition of Effects in Path Analysis". American Sociological Review, 40(1): 37-47.

[6] Anand, P (2016). Happiness Explained. Oxford: Oxford University Press

[7] Baetz, M. and Toews, J. (2009). "Clinical implications of research on religion, spirituality, and mental health". Canadian Journal of Psychiatry. 54 (5): 292-301.

[8] Barro, R. J., and Lee J.W., (2001). "International data on educational attainment: Updates and implications". Oxford Economic Papers 53 (3): 541-563.

[9] Bjork, G.J., (1999). The Way It Worked and Why It Won't: Structural Change and the Slowdown of U.S. Economic Growth. Westport, CT; London: Praeger. pp.: 251, ISBN 0-275-96532-5.

[10] Deci, E. L. and Ryan, R. M. (2006). "Hedonia, Eudaimonia, and Well-being: An introduction". Journal of Happiness Studies 9 (1): 1-11. Doi:10.1007/s10902006-9018-1

[11]Dickinson, E., (2012). "GDP: a Brief History”, ForeignPolicy.com, and Retrieved 25 April 2012.

[12] Dodge, Y. (2003). The Oxford Dictionary of Statistical Terms. OUP. ISBN 0-19-920613-9.

[13] Dunn, E. W., Aknin, L. B. and Norton, M. I. (2008). "Spending Money on Others Promotes Happiness". Science. $\quad 319 \quad$ (5870): $1687-8$. Doi:10.1126/science. 1150952

[14]Ellison, C.G. and George, L.K. (1994). "Religious Involvement, Social Ties, and Social Support in a Southeastern Community". Journal for the Scientific Study of Religion. 33 (1): 46-61. Doi:10.2307/1386636. JSTOR 1386636.

[15]Frey, B. S. and Stutzer, A. (2001). Happiness and Economics. Princeton: Princeton University Press. ISBN 0-691-06998-0.

[16] Frey, B. S. (2011). "Happy People Live Longer". Science. 331 (6017): 542-3.

Doi:10.1126/science. 1201060
[17] Galor, O. (2005). From Stagnation to Growth: Unified Growth Theory. Handbook of Economic Growth. Elsevier.

[18] Graham, M. C., (2014). Facts of Life: Ten Issues of Contentment. Outskirts Press. pp. 6-10. ISBN 978-14787-2259-5.

[19] Helliwell, J., Layard, R. and Sachs, J. (2016). "World Happiness Report". United Nations Development Programme.

[20] Helpman, E., (2004).The Mystery of Economic Growth. Harvard: Harvard University Press.

[21] Hornby, A.S., (1985). Oxford Advanced Learner's Dictionary of Current English, Twenty Second Impression, Oxford: Oxford University Press.

[22] Human Development at https://en.wikipedia.org/wiki/Human_development_(hu manity).

[23]IMF. (2012). "Statistics on the Growth of the Global Gross Domestic Product (GDP) from 2003 to 2013".

[24] Nussbaum, M. (2000). Women and human development: the capabilities approach. Cambridge New York: Cambridge University Press. ISBN 9780521003858.

[25] Nussbaum, M. (2011). Creating Capabilities: The Human Development Approach. Cambridge, MA: Harvard University Press. pp. 3334. ISBN 0674072359.

[26] Routledge, C. (2012).”Are Religious People Happier Than Non-religious People?" Psychology Today. Retrieved 24 January 2014.

[27] Sen, A. (2005). "Human Rights and Capabilities". Journal of Human Development. 6 (2): 151-166. Doi:10.1080/14649880500120491.

[28] Solow, R. M., (1956). "A Contribution to the Theory of Economic Growth”. Quarterly Journal of Economics. 70 (1): 65-94.

[29] Srinivasan, T.N. (1994). "Human Development: A New Paradigm or Reinvention of the Wheel?" Human Development 84 (2): 238-243.

[30] Steptoe, A., Wardle, J. and Marmot, M. (2005). "Positive affect and health-related neuroendocrine, cardiovascular, and inflammatory processes". Proceedings of the National Academy of Sciences. 102(18): 6508-12. Bibcode:2005PNAS..102.6508S. Doi:10.1073/pnas.0409174102.

[31] Streeten, P. (1994). "Human Development: Means and Ends". Human Development, 84(2): 232-237.

[32] Swan, T.W., (1956). "Economic Growth and Capital Accumulation”. Economic Record, 32: 334-361. Doi: 10.1111/j.1475-4932.1956.tb00434.x.

[33]Ul-Haq, M. (2003). "The Human Development Paradigm". In Fukuda-Parr and A. K. Shiva Kuma, A.K.S. Eds. Readings in Human Development, 1734.Oxford, UK: Oxford University Press.

[34] United Nations Development Programme, 2015 , "Human Development Reports: at http: http://hdr.undp.org/en/2015-report.

[35] United Nations Development Programme, 2015, "Human Development Reports" at http://hdr.undp.org/eandevn/hum

[36] United Nations Development Programme. (1997). "Human Development Report". pp: 15. ISBN 978-0-19511996-1 
[37] United Nations Development Programme. (2016).’Human Development Report 2015: Work for Human Development" Web Version available at http://hdr.undp.org/en/data.

[38] World Bank. (2016). "Annual Gross Domestic Product Growth (\%)". Available on line at http://data.worldbank.org/indicator/NY.GDP.MKTP.KD .ZG.

[39] World Health Organization. (2016). "Preventing disease through healthy environments: a global assessment of the burden of disease from environmental risks". Available at http://www.eho.org/ Retrieved 4 August 2016

[40] Wright, S. (1921). “Correlation and Causation". Journal Agricultural Research. 20, 557-585.

[41] Wright, S. (1934). "The Method of Path Coefficients". Annals of Mathematical Statistics, 5(3), 161-215.

\section{Author Profile}

Muchdie is a $\mathrm{PhD}$ holder in Economics from Department of Economics at the University of Queensland, Australia. He had been a senior researcher and Director at the Agency for the Assessment and Application of Technology, the Government of Indonesia (19792010) before he moved to Universitas Muhammadiyah Prof. DR. HAMKA as Senior Lecturer in 2010. He had been the Head of Management Department at Post Graduate School (2009-2010) before appointed as Vice Rector for Academic Affair at Universitas Muhammadiyah Prof. DR. HAMKA (2010-2014). He is now serving as Vice Rector for Administrative and Financial Affair at Universitas Muhammadiyah Prof. DR. HAMKA. 